\title{
L'HOMME L'Homme
}

$218 \mid 2016$

Varia

\section{La redescription de l'anthropologie selon Marilyn Strathern}

Marilyn Strathern's Redescription of Anthropology

\section{Ashley Lebner}

Traducteur : Arianne Dorval

\section{Q OpenEdition \\ Journals}

Édition électronique

URL : http://journals.openedition.org/lhomme/28946

DOI : 10.4000/lhomme.28946

ISSN : $1953-8103$

Éditeur

Éditions de l'EHESS

Édition imprimée

Date de publication : 20 mai 2016

Pagination : 117-149

ISSN : 0439-4216

Référence électronique

Ashley Lebner, "La redescription de l'anthropologie selon Marilyn Strathern », L'Homme [En ligne],

218 | 2016, mis en ligne le 19 mai 2018, consulté le 01 mai 2019. URL : http://

journals.openedition.org/lhomme/28946; DOI : 10.4000/lhomme.28946

(c) École des hautes études en sciences sociales 


\title{
La redescription de l'anthropologie selon Marilyn Strathern
}

\author{
Ashley Lebner
}

M

ARILYN Strathern peut tout à fait commencer un essai par une vignette ethnographique et y répondre par une autre, préférant cultiver l'aspect énigmatique de son objet plutôt que d'en donner la clé. Chez Strathern, la forme fait bien sûr partie de l'argument, ce qui est une manière pour elle d'attirer ses lecteurs, dans l'espoir de capter leur attention et non de les obliger à respecter les termes d'un contrat. Cette transformation de la forme et de l'argumentation, cette façon d'écrire avec et à partir des récits des autres, est une facette de ce que j'appellerai ici "redescription ", bien qu'une redescription implique aussi de mettre en œuvre d'autres relations, comme nous le verrons plus loin ${ }^{1}$. Même si elle "dissimule" certains termes de son argumentation, Strathern choisit ses mots et ses formes très

1. Dans un texte plus ancien, j'ai proposé une interprétation embryonnaire de la "redescription ", en réaction à la Huxley Memorial Lecture prononcée par Marilyn Strathern (Strathern 2006 ; Lebner \& Deiringer 2009: 2).

Cet essai a d'abord été présenté au séminaire d'anthropologie socioculturelle de l'Université du Michigan, où il a bénéficié des commentaires précieux de Stuart Kirsch, Elizabeth Roberts et Erik Mueggler. Je tiens à remercier tout particulièrement ce dernier pour les conseils qu'il m’a donné aux étapes critiques de son élaboration, mais aussi lors des nombreuses discussions qui ont accompagné son écriture. Les commentaires d'Alberto Corsín-Jiménez ont suscité une réflexion et des encouragements bienvenus, et ceux de Casper Bruun Jensen ont laissé des traces précieuses. Les évaluateurs anonymes ont posé de bonnes questions, me poussant à développer davantage mes réponses. Olivier Allard a fourni une aide inestimable pour la publication du texte en français, tout comme le comité de rédaction de L'Homme et, bien sûr, la Faculté des arts de l'Université Wilfrid Laurier, qui a généreusement octroyé les fonds ayant permis la traduction soignée d'Arianne Dorval. Je remercie également Berghahn Books d'avoir autorisé la traduction de ce texte, dont la version anglaise sera en partie publiée dans l'introduction d'un ouvrage collectif à paraitre, intitulé : Redescribing Relations: Strathernian Conversations on Ethnography, Knowledge and Politics. Enfin, je tiens à exprimer ma plus profonde gratitude à Marilyn Strathern pour le soutien indéfectible qu'elle m'a apporté tout au long du projet plus vaste qui a donné naissance à cet essai, pour ses propos encourageants à l'égard d'une version antérieure du texte et, surtout, pour le bonheur que m’a toujours procuré la lecture de son œuvre. 
soigneusement - ce n'est pas toujours manifeste, son écriture étant souvent jugée simplement " difficile». En effet, le plus important lorsqu'on entame la lecture de l'œuvre de Strathern, voire d'un texte sur Strathern, est sans doute ceci : prêter attention aux mots et aux formes, ainsi qu'aux relations entre ceux-ci - relations qui comprennent, bien évidemment, les distinctions.

Mary Douglas a dit un jour de Strathern qu'elle était une " anthropologue d'anthropologue» (ce qui n'était pas vraiment une flatterie) ${ }^{2}$. Si le travail d'envergure qu'elle a accompli suscite aujourd'hui de l'intérêt dans l'ensemble des sciences humaines et sociales ${ }^{3}$, Strathern n'en est pas pour autant toujours bien comprise. Néanmoins, et peut-être même parfois du fait de cette mauvaise compréhension, elle est souvent considérée comme l'une des plus éminentes "théoriciennes" de l'anthropologie, dont le travail ne pourrait être réduit à un seul concept, un seul thème ou un seul principe. C'est sans doute vrai, jusqu'à un certain point. Strathern a effectivement écrit sur un nombre impressionnant de sujets, dans les domaines de la parenté, du sexe, de la science, du droit, de l'économie et de la bureaucratie. En outre, elle a fait évoluer petit à petit, au fil du temps, son répertoire de mots et d'images (mais pas les enseignements qu'elle a tirés en les élaborant). Pourtant, comme elle le fit au cours d'un récent entretien, Strathern insiste sur le fait que son projet est moins de développer une théorie ou des concepts que de produire une "bonne description" (Borič 2010), de faire de la «bonne» ethnographie (Edwards \& PetrovičŠteger 2011 ; Allard 2014 ; Street \& Copeman 2014). En d'autres termes, le projet de Strathern associe le rejet de la «théorie » à un but singulier, qui implique un travail permanent, puisqu'à ses yeux aucune description n'est jamais parfaite ni définitive. Dans la mesure où, pour Strathern, "une discipline n'est ni plus ni moins que l'effort de décrire, [et où] le génie de l'anthropologie a toujours été son engagement descriptif avec l'acte même de description, avec la façon dont les gens produisent des récits d'euxmêmes » (2005a : XII), nous comprenons pourquoi le mot "redescription » est celui qui rend le mieux compte de son approche de l'anthropologie.

Cela étant, la redescription strathernienne se veut aussi beaucoup plus critique, voire politique, qu'on ne pourrait le supposer en la réduisant à une "re-constitution cohérente de récits». Elle est critique, bien sûr, en partie parce qu'elle renvoie à la manière dont Strathern reformule le sens de la critique dans la recherche, à savoir : "multiplier et diviser à

2. On retrouve ce commentaire dans une brève biographie de Strathern qui apparaît sur le site Cultural Antropology : http ://www.culanth.org/articles/347-intervening.

3. Strathern a suscité l'intérêt de chercheurs dans diverses disciplines, depuis la comptabilité, le commerce, la littérature anglaise jusqu'à la géographie, la philosophie et la sociologie/science and technology studies (soit, respectivement, Michael Power, Paul du Gay, Mary Jacobus, Nigel Thrift, Isabelle Stengers, Rolland Munro et Bruno Latour, pour ne nommer que ceux-là). 
nouveau les résultats de toute thèse particulière " (2006 : 199). Or, à cette idée de la critique s'ajoute une attention constante au langage analytique et à la façon dont celui-ci peut être décentré. Comme Strathern le sait pertinemment, le langage analytique conventionnel a joué un rôle pernicieux dans le discours de l'État, que ce soit intentionnellement ou non (Strathern 1996a; Greenhouse 2017). S’il est vrai qu'elle a peu écrit sur la "politique» en tant que telle, les interprétations qui en déduisent que son œuvre est apolitique (par exemple, Josephides 1991) passent à côté de ce que l'écriture de Strathern nous dit: la manière la plus stratégique de mener une "politique" anthropologique est de modifier les mots et les formes au travers desquels s'exprime l'anthropologie.

Avant d'examiner la politique inhérente à la redescription selon Strathern, cet essai s'attachera à élucider une série de propositions imbriquées. Il s'agira, en effet, non pas de retracer l'utilisation répétée d'un " concept ", mais d'appréhender la redescription comme une série de relations. Et cela, non seulement parce que Strathern emploie le mot et ses variantes - telles que "redécrire " - avec parcimonie dans son œuvre ${ }^{4}$, mais aussi, et de façon encore plus significative, parce que l'anthropologie de Strathern ne privilégie pas la création de " concepts".

"L'invention de concepts" a longtemps été associée au travail de la philosophie ${ }^{5}$. Or, dans la mesure où Strathern a pour projet de mettre en lumière la contribution spécifique de l'anthropologie, et en particulier de l'ethnographie, elle se méfie, comme nous le verrons bientôt, de l'utilisation des catégories analytiques usuelles, telles que "la société », "l'individu ", «la comparaison " (au sens conventionnel) et, plus généralement, «la théorie». En revanche, elle ne s'oppose jamais frontalement à la notion de "concept", ce qui paraît difficile compte tenu des contraintes du langage universitaire. Et pourtant, on ne peut négliger l'importance de la série de "relations " qui stimule ses analyses. Si Strathern définit les relations comme les connexions et les distinctions conceptuelles et interpersonnelles qui constituent la vie sociale (Strathern 2005b:9-14), il convient de souligner qu'à ses yeux chaque mot compte: bien que les concepts soient relationnels, orienter l'anthropologie vers la création de concepts n'est pas la même chose que d'" utiliser des relations pour dévoiler des relations" (Ibid.: VII). Cette dernière proposition, qui est

4. En outre, Strathern n'utilise pas le terme "redescription" uniquement pour se référer à son propre travail. En plus de l'employer pour désigner ce qu'elle fait, et ce, même dans ses travaux les plus récents (par exemple, Strathern 2011a et b, 2014a), elle y a recours parfois dans ses observations critiques de ce que d'autres font - et ne devraient peut-être pas faire - avec des concepts spécifiques (par exemple, Strathern 2003b).

5. Voir, en particulier, Gilles Deleuze \& Félix Guattari (1991), qui ont inspiré une série de réflexions anthropologiques stimulantes. 
une autre des définitions de l'anthropologie formulées par Strathern, doit être comprise comme une réponse à l'appel lancé aux anthropologues pour qu'ils et elles commencent à créer des concepts. La proposition de Strathern indique aussi déjà pourquoi son œuvre ne saurait être assimilée au tournant ontologique, sur lequel je reviendrai plus tard, même si elle apprécie la créativité de ses principaux instigateurs ainsi que leur souci de rendre hommage à la différence.

Dans ce qui suit, la redescription sera examinée afin d'éclairer la façon dont les relations animent l'anthropologie de Strathern. Élaborée tout au long de son ouvre de maturité, la redescription a d'abord été formulée dans The Gender of the Gift (1988), livre qui "désécrit " son premier ouvrage, Women in Between (Strathern 1972, voir aussi 2017). Dans une section clé de l'introduction de The Gender of the Gift, intitulée «Negativities : Redescribing Melanesian Society» (1988: 11), Strathern expose l'idée-force de sa contribution. Elle va plus loin que dans ses travaux antérieurs fondés sur la "mise en place de négativités " (Ibid.), où elle démontrait que différents concepts - tels que "nature " et "culture» (Strathern 1980), ou encore « droit » et " contrôle social » (Strathern 1985) ne pouvaient s'appliquer au cas de Hagen, principal terrain de son enquête ethnographique dans les montagnes de la Papouasie-Nouvelle-Guinée. The Gender of the Gift aborde plutôt la question plus large de la description elle-même: "Nos propres métaphores traduisent une métaphysique profondément enracinée, qui se manifeste dans toutes sortes d'analyses. La question est de savoir comment les décentrer le plus efficacement possible» (1988: 12). En décentrant certains concepts clés - en particulier ceux de "société" et d' "individu"-, de nouvelles relations analytiques sont élaborées dans The Gender of the Gift, dans Partial Connections (2004 [1991]) et tout au long de son ouvre ultérieure : depuis le déploiement de l'analogie jusqu'à la découverte de relations qui remettent en cause la notion d'échelle. On peut dire qu'il s'agit de relations car elles opèrent à partir d'autres termes. Elles constituent ainsi le cour de la redescription (elle-même bien sûr une «re »lation), rendant possible un nouveau mode de critique et de politique anthropologique.

Décentrement, analogie, relations, politique: j'examinerai chacune de ces relations à tour de rôle pour montrer comment elles sous-tendent les redescriptions qui sont au cœur de l'anthropologie de Strathern. Autrement dit, je m'efforcerai d'établir que chacune de ces relations - décentrement, analogie, relations, politique - est imbriquée dans l'autre, qu'ensemble, elles constituent les coordonnées de base de l'écriture de Strathern. Il ne s'agit pas de prétendre qu'on ne peut aborder séparément les différentes parties de son œuvre. Néanmoins, privilégier l'unité de la 
pensée de Strathern me semble représenter la meilleure façon d'aider de nouveaux lecteurs à y entrer. Par ailleurs, si mon objectif premier n'est aucunement de répondre aux critiques ou de les contester, j'ai le sentiment que nombre de jugements portés sur le travail de Strathern n'ont pas tenu compte de son projet relationnel plus vaste, qui est de redécrire l'écriture ethnographique, tout autant que l'anthropologue comme auteur(e) - voire comme personne -, à partir des relations qui le ou la composent. Ainsi Strathern crée-t-elle un nouveau genre ethnographique, bien qu'elle n'envisage pas que d'autres puissent le suivre tel quel, compte tenu de leurs propres implications relationnelles.

Si Strathern ne propose pas de "programme» à reproduire ${ }^{6}$, cela ne signifie pas pour autant que son projet soit "aléatoire». Au contraire, comme elle le dit elle-même, "j'espère qu'il comporte une certaine cohérence" (Borič 2010 : 281). Le but de cet article est de montrer que c'est incontestablement le cas: depuis The Gender of the Gift, Strathern a développé un projet remarquablement cohérent qui offre des réponses aux nombreux arguments qu'on lui oppose - y compris aux arguments politiques. Que ces réponses soient satisfaisantes ou non est une question que d'autres pourront poser plus tard; notre première tâche est néanmoins de bien les comprendre. Si je m'adresse ici surtout aux anthropologues, c'est que la redescription par l'ethnographie à laquelle s'est attelée Strathern les interpelle en premier lieu: son projet invite tout chercheur qui le veut bien à redécrire des relations, ce qui lui apporterait une compréhension nouvelle des diverses pratiques de savoir, mais aussi lui permettrait d'exercer le rôle critique, voire même "politique", auquel il ou elle pourrait prétendre.

\section{Commencer par décentrer}

La redescription commence en décentrant des concepts. Pour Strathern, c'est bien par là qu'il faut commencer, car certains concepts euro-américains sont si dominants qu'ils corrompent toute description. Aussi les anthropologues doivent-ils décentrer ces concepts afin de "créer des espaces qui [...] manquent aux analyses exogènes" (1988: 11). Or, cet acte implique une série d'autres gestes analytiques qui surgissent en partie des concepts ainsi décentrés. Cela s'applique particulièrement aux principaux termes que décentre Strathern dans The Gender of the Gift et dans tous ses travaux ultérieurs : la "société » et son compagnon naturel, l'«individu ».

6. Strathern associe le fait d'avoir un programme à la conception d'une "fin globale " plutôt totalitaire qui doit être suivie par d'autres (Borič 2010 : 281). Voir aussi Lisette Josephides, Nigel Rapport \& Marilyn Strathern (2015 : 399). 
Il convient de s'attarder un instant sur ce premier décentrement, car on note rarement jusqu'à quel point il est au cœur même de l'anthropologie de Strathern. En effet, on rencontre souvent l'idée qu'elle l'aurait accompli uniquement dans The Gender of the Gift. Or, il est au fondement de la créativité analytique de toute son œuvre. Comme l'explique Strathern, si ce décentrement s'avère nécessaire, ce n'est pas parce que "société " et « individu » constituent de mauvaises traductions de concepts indigènes. En fait, pour Strathern, la traduction n'est jamais qu'une "illusion" (Ibid.: 29); on ne peut pas rendre les autres parfaitement lisibles, tels qu'ils "sont", par le biais du langage anthropologique. Les anthropologues peuvent espérer produire de meilleures descriptions uniquement grâce à une vigilance constante et après avoir d'abord traité de l'opposition perçue entre société et individu. C'est que cette opposition est particulièrement tenace: "La société est envisagée comme ce qui relie les individus les uns aux autres, soit les relations entre eux. On conçoit ainsi la société comme ce qui ordonne et qui classe et, en ce sens, comme une force unificatrice » (Ibid.: 12). Cette force est censée réunir et façonner des individus uniques, qui peuvent ensuite transformer cette relation - bien que les individus soient toujours, fondamentalement, «imaginés comme étant conceptuellement distincts des relations qui les rassemblent " (Ibid. : 13). C'est cette notion persistante de société et d'individu, d'un ensemble composé de parties individualisées mais qui les englobe à la fois, que Strathern décortique pour arriver à sa première description de ce qu'elle nomme la "socialité mélanésienne ». La Mélanésie, il faut le préciser, est moins un lieu qu'une synthèse ethnographique et une expérience qui explore la possibilité de penser la différence dans le cadre de l'analyse euro-américaine (à noter que mes futures références à la Mélanésie seront en réalité à la « Mélanésie »; Gell 1999). À l'inverse, la "socialité", définie par le fait de «créer et maintenir des relations» (Ibid.: 13), permet d'étudier la Mélanésie loin de l'ombre portée par ce qu'on pourrait appeler la "pensée-société ». Celle-ci est encore plus persistante et sans doute moins bien comprise que ne l'est la "pensée-marchandise " (l'hypothèse selon laquelle les individus different des «choses" et sont moins aliénables). Pourtant, et c'est crucial, la pensée-société englobe la pensée-marchandise, voire la génère ${ }^{7}$.

7. La pensée-marchandise implique un ensemble d'hypothèses selon lesquelles les personnes individuelles sont des sujets différents des choses ou des objets, les premières pouvant agir, posséder et aliéner la propriété qu'elles ont d'elles-mêmes ou de choses (mais non d'autres personnes). La critique formulée par Strathern de la pensée-marchandise a suscité une grande attention; on retrouve cette volonté de relativiser la distinction entre sujet et objet bien au-delà de l'anthropologie, et surtout dans les travaux de Bruno Latour. De ce fait, et parce que la critique qu'effectue Strathern de la société englobe, comprend et va au-delà de sa critique de la pensée-marchandise, je me concentrerai sur la première. 
Bien sûr, décentrer la société et l'individu revient à déstabiliser les concepts fondateurs de l'anthropologie moderne. La «société » fut d'abord prédominante au sein de l'anthropologie sociale britannique, et ce, dès ses débuts fonctionnalistes. Certes, l'anthropologie sociale classique a souvent enjoint à ses praticiens de ne pas "réifier" la société ; elle a également privilégié la " personne " plutôt que l'individu, soulignant le fait que celleci "était déjà un élément d'une relation sociale, déjà [...] une fonction de la relation", un relatum ${ }^{8}$ (Strathern 2005b : 41). Une telle précédence accordée aux relations explique aussi pourquoi la personne est un concept essentiel pour Strathern, et pourquoi elle est plus redevable à l'anthropologie sociale classique qu'au structuralisme (Strathern 2017) ${ }^{9}$ - une autre raison pour laquelle les lecteurs français devraient cesser de croire que son anthropologie n'est qu'une forme de structuralisme déguisé. Pourtant, les anthropologues sociaux étaient encore attachés à la société : ils étaient soucieux de décrire des "systèmes formant une "unité complexe" ou, plus généralement [...], une ou des structure[s], qui constituai[en]t "un arrangement de personnes dans un système institutionnel de relations déterminées" "(Radcliffe-Brown 1952: 53 ; cité in Strathern 2005: 41). Même si les anthropologues sociaux ont pu faire preuve d'une certaine réflexivité concernant les problèmes que la société et l'individu posaient à l'ethnographie, ils réinstituèrent donc ces derniers par le biais de «systèmes » et de "structures ».

La société était également présente dans des modèles postérieurs, perçus à leur époque comme des outils de transformation ou de modernisation de l'anthropologie (sociale), et c'est en ce sens qu'elle est décentrée par Strathern. Par exemple, dans The Gender of the Gift (1988), Strathern s'intéresse au féminisme et, par association, à la tradition marxiste dont il s'inspire fréquemment. Le marxisme a longtemps influencé les traditions critiques parce qu'il joint le juridictionnel à l'analytique : il théorise la production des inégalités tout en exposant les stratégies de classe qui conduisent à la domination et à l'exploitation. Quoique le féminisme (ou certaines " approches » féministes) ait pu critiquer le marxisme du fait de son androcentrisme, il n'en partage pas moins avec lui un intérêt pour l'étude des formes d'inégalité et pour la lutte contre celles-ci (mais en se

8. Je remercie Marilyn Strathern d'avoir corrigé la citation originale.

9. Il est vrai que la synthèse ethnographique de la Mélanésie effectuée par Strathern fait écho au structuralisme de Claude Lévi-Strauss (en particulier aux Mythologiques), dans la mesure où les différences culturelles y sont présentées comme étant des versions les unes des autres. Cependant, comme elle l'affirme dans un entretien à paraître (Strathern 2017), le structuralisme lui a servi comme technique plutôt que comme théorie. En outre, l'anthropologie sociale classique ayant elle aussi élaboré une pensée solide de la relation, Strathern traite dans ses écrits de l'influence qu'a exercée sur elle l'anthropologie sociale (cf., par exemple, Strathern 1995). 
référant spécifiquement au genre). Tout en sympathisant avec ces approches - Strathern est féministe et a été l'une des premières anthropologues à écrire spécifiquement sur les femmes (Strathern 1972) -, elle s'interroge sur l'effet que peuvent avoir de telles analyses critiques sur l'ethnographie. En effet, bien que ces analyses s'intéressent aux relations, le mot "inégalité » évoque avant tout un cadre sociétal global avec sa division en groupes - des classes et des sexes -, qui sont constitués à leur tour d'agents individuels. En outre, l'inégalité nous pousse à concevoir la différence comme étant en fin de compte toujours hiérarchique : à échelles multiples (Strathern 1987a, 1987b : 287). Pourtant, avant même que Strathern ne songe à critiquer la société - et son produit conceptuel qu'est l'«inégalité»-, elle écrivait à propos des préoccupations féministes :

«[...] des notions telles que biais masculin ou point de vue féminin peuvent être extrêmement fructueuses, et certainement transformer notre façon de "voir" [...]. Néanmoins, le bruit de notre propre labeur ne doit pas nous assourdir au point de nous faire oublier que les autres sont eux aussi créatifs» (1981 : 684).

Il s'agit là d'une des critiques les plus acerbes et les plus directes qu'elle ait formulées par écrit ${ }^{10}$ à l'encontre d'autres anthropologues ou écoles de pensée. Or, cette critique signale aussi le début d'un malaise persistant face au féminisme et à différentes modes intellectuelles qui ont fait peser les préoccupations euro-américaines directement sur l'ethnographie (Strathern 1987c). Si la pratique critique de Strathern a évolué au cours de l'élaboration de son approche redescriptive, durant les années 1980, son souci de faire place à la créativité des autres n'a cessé d'orienter ses efforts depuis lors.

C'est peut-être la priorité accordée à l'ethnographie dans son œuvre qui a conduit ses détracteurs à méconnaitre la démarche politique de Strathern, alors même que décentrer la société avait une réelle portée critique dans le contexte de la révolution néolibérale (toujours en cours) - laquelle s'incarne dans la célèbre phrase prononcée par Margaret Thatcher en 1987 : "Il n’y a pas de société " (cité in Strathern 1996a : 53). Je reviendrai plus longuement sur cette question dans la quatrième partie de cet essai. Mais la "politique» de Strathern, ainsi que son intérêt critique pour l'anthropologie à l'égard du pouvoir sont pleinement intégrés à sa pratique redescriptive. Je me pencherai donc tout d'abord sur les propositions analytiques qui constituent la redescription elle-même.

Jusqu’à présent, je me suis concentrée sur l'acte de décentrer. Plutôt que de codifier un concept dans le répertoire de Strathern, je voulais éclairer sa pratique : d'abord, l'élimination permanente des concepts de "société"

10. Strathern répondait ici à l'anthropologue Annette Weiner, qui l'avait accusée d'écrire d'un point de vue masculin. Voir l'entretien de Strathern avec Eduardo Viveiros de Castro et Carlos Fausto (Strathern 2017). 
et d' «individu» de son travail ${ }^{11}$ et, ensuite, une tendance plus générale à éviter les concepts euro-américains surdéterminants, qui sont souvent associés d'emblée à la société. En effet, depuis The Gender of the Gift, Strathern observe la façon dont la société hante les modèles anthropologiques qui se sont succédé. Partial Connections (2004 [1991]), sur lequel je reviendrai en détail plus loin, montre ainsi comment une multitude de tentatives de rethéorisation de la discipline, à la fin des années 1980, ont réinstitué la pensée-société - depuis la "crise de la représentation » et les théories néo-marxistes de la mondialisation jusqu'à l'anthropologie comparative. Plus précisément, ces tentatives mobilisent les images d'une échelle ou d'une abstraction qui transcende les cas individuels concrets (par exemple, global/local ou théorie/donnée), ou bien décrivent comment un tout se fragmente en plusieurs parties (par exemple, la condition postmoderne). Dans After Nature (1992a), qui traite de la parenté en Angleterre avant et après l'arrivée de la procréation médicalement assistée, Strathern montre que le constructivisme social réinstitue la société de façon similaire, puisqu'on y voit se co-construire des forces sociétales/ collectives/discursives et des forces individuelles. Le constructivisme est dès lors une approche tout aussi "pluraliste» et "mérographique», et donc "moderne", que ces pratiques de savoir qu’il prétend avoir abandonnées - et d'ailleurs, comme Alberto Corsín-Jiménez (2015: 184) en conviendrait sans doute, le constructivisme a développé son propre mode de redescription ${ }^{12}$. Strathern se penchera plus tard sur les études en Sciences, technologies et société (ci-après STS) et même sur Derrida dans Property, Substance and Effect (Strathern 1999a et b), afin de souligner

11. C'est surtout dans le cadre de ses ethnographies de l'Angleterre et du Royaume-Uni qu'elle parle de la société et de l'individu comme d'objets ethnographiques.

12. Dans After Nature, le pluralisme renvoie à l'idée euro-américaine selon laquelle le monde est composé d'une "pluralité" de formes individuelles pouvant être rassemblées en groupes: humains/personnes, animaux, sociétés. Sa notion de relation "mérographique " appréhende d'autres aspects de cette vision plurielle, à savoir que chacune de ces formes peut être décrite comme faisant partie des autres, qui lui servent de "contextes", et n'est ainsi jamais pareille aux autres (1992a: 72-81). Le pluralisme et les relations mérographiques sont en définitive liés à la pensée-société; en effet, bien qu'il soit techniquement plus vaste, le concept de connexion mérographique est calqué sur la pensée-société. D'ailleurs, comme l'affirme Strathern, les différentes formes de classification sont "présupposées dans la connexion mérographique indigène (anglaise) entre l'individu et la société. On peut changer de perspective en passant d'une entité à l'autre, de sorte que les deux perspectives semblent englober à elles deux tout ce qui peut être dit à propos de la vie sociale» (Ibid.: 76). Je sais fort bien que les constructivistes sociaux ne se définissent pas tous comme postmoderne; or, à l'époque d'After Nature, constructivisme et postmodernisme étaient devenus de plus en plus synonymes. After Nature est une tentative de définir la période "postplurielle " émergente, qui se caractérise moins par l'interrogation de la vérité que par la reconnaissance de la contingence d'une appréhension plurielle de la réalité. Plus récemment, Strathern a dit de ce pluralisme qu'il est un "perspectivalisme ", lequel perdure encore dans certains travaux en STS (à ne pas confondre avec le perspectivisme, cf. Strathern 2011a). 
comment ceux-ci réintroduisent clandestinement la société via le concept de l'infini ("les réseaux infinis" des STS et "la conception grammatologique des équations récurrentes » chez Derrida) ${ }^{13}$. Et la pensée-société hante sans doute les récentes discussions sur l'ontologie, même si Strathern met l'accent dans ses écrits sur d'autres soucis qu'elle a avec ce concept. J'y reviens dans la troisième partie (voir notamment la note 17).

En somme, Strathern met constamment en lumière le fait que la "société " est non seulement un terme, mais aussi une façon de penser qui imprègne toutes nos approches analytiques et qui obscurcit l'ethnographie avec une mathématique ou une notion d'échelle euro-américaine. À l'inverse, on relève rarement que tout l'intérêt de Strathern pour la notion d'échelle et la subversion qu'elle en opère sont dus au fait que cette notion obsède les analyses euro-américaines à travers la pensée-société : à savoir la hiérarchie et l'englobement qu'évoquent la société (plus grande) et les individus (plus petits). Ce qui nous amène au problème de la comparaison.

\section{Comparaison, (comme) analogie}

La comparaison est souvent considérée comme un concept stable au cœur de l'anthropologie de Strathern. Or, ce n'est pas tout à fait exact. Si Partial Connections s'attelle précisément à "évacuer le problème de la "comparaison" " (2004 [1991] : XXVIII), il est clair que The Gender of the Gift s'y était déjà attaqué avant. Il convient ici de citer à titre indicatif les objectifs de redescription qui sont au centre de ce livre:

"Je décentre ce que "nous" pensons être la société par un ensemble de constructions différentes, mises en avant par opposition afin de suggérer une analogie avec "leur" vision. Dans le même temps, cette analogie perçue comme comparaison, qui aborde les deux ensembles d'idées comme des préceptes pour l'action sociale, élargit alors pour nous le sens originel du concept» $(1988: 17)$.

Si cette dernière phrase résume à elle seule toutes les manœuvres analytiques qu'effectuera Strathern pour arriver à sa redescription de la société mélanésienne, elle est aussi une affirmation générale du fait que c'est en décentrant que l'on produit de nouvelles relations conceptuelles - en particulier l'analogie, qu'elle oppose à la comparaison. Strathern concède que son analogie sera "appréhendée " comme une comparaison.

13. «Comme je l'ai déjà suggéré, la société, au sens où on l'entendait au XX⿳亠口冋丁 siècle dans la sphère euro-américaine, est déjà la preuve de telles conceptualisations de l'infini. Il est dit qu'elle porte en elle la diversité, qu'elle est constituée de différents sujets dénombrables/innombrables, chacun ayant son propre point de vue - que ces sujets soient des institutions, des groupes, des catégories ou des personnes individuelles" (1999b : 237). 
Et pourtant, elle se distancie nettement de cette dernière, voire sans doute plus généralement $\mathrm{du}$ "concept " - un terme bien sûr ordinairement conçu pour faciliter l'entreprise comparative et même souvent universalisante de la philosophie. Ce qui précède suggère en effet qu'avec l'analogie, qui fait partie d'un autre ensemble de "constructions", ce n'est pas seulement le sens de la société qui est élargi, mais aussi la comparaison (de même que le «concept»). En d'autres termes, en plus de décentrer et d'élargir la société, il faut pratiquer la comparaison autrement. Strathern propose l'analogie.

C'est dans Partial Connections, un livre centré sur son approche de l'écriture, que Strathern montre que la comparaison, telle qu'elle est ordinairement conçue, dérive de la pensée-société qu'elle s'efforce d'éviter. Bien sûr, la critique de la comparaison remet en cause l'anthropologie tout autant que le décentrement de la société - car, après tout, la comparaison est au cœur de cette discipline depuis le XIX siècle. Certes, les pratiques comparatives ont évolué. Les "évolutionnistes en chambre" (armchair evolutionists), comme Morgan, Tylor et Frazer, ont d'abord extrait des traits sociaux spécifiques de leur contexte afin de les comparer (Strathern 1987d : 265). Puis, les chercheurs de terrain du début du XXe siècle ont étudié les structures de sociétés distinctes, en espérant que des comparaisons futures en tireraient des lois sociales universelles. De nombreux projets ont reformulé cet objectif vers le milieu du XXe siècle: néo-évolutionnisme, structuralisme et études holoculturelles ${ }^{14}$ (Gingrich $\&$ Fox 2002). Au-delà de ces projets comparatifs plus explicites, et même quand la méthode comparative semblait "impossible", celle-ci était encore souvent considérée comme la "seule méthode en anthropologie " - comme l'aurait, paraît-il, affirmé Evans-Pritchard (Needham 1975: 365) ${ }^{15}$. Cette foi inébranlable en la comparaison, qui a perduré jusqu'à la fin des années 1980, peut expliquer pourquoi les spécialistes de la Mélanésie, entre autres, ont continué à pratiquer la comparaison en dépit de nombreux problèmes ${ }^{16}$. Quelles sont les unités d'analyse appropriées pour la comparaison? Comment les frontières sont-elles établies? Strathern montre que les solutions proposées reposent toutes sur une mathématique connue : des "cas individuels" (sociétés ou traits sociaux) sont comptés

14. Les études holoculturelles étaient représentées par le Human Relations Area Files.

15. Certes, les articles écrits par des ethnographes engagés, formés autour du milieu du siècle, contredisent une certaine perception selon laquelle la comparaison/généralisation était un «objectif supérieur » en anthropologie (cf. Pitt-Rivers 1974).

16. La comparaison en Mélanésie promettait d'expliquer comment les sociétés pouvaient être liées entre elles, comment et pourquoi elles se transformaient et, même, comment elles évoluaient d'une «forme» vers une autre (Strathern 2004 [1991]). 
et évalués par une "entité" (la "théorie») de plus grande dimension.

C'est ce procédé qui permet de découvrir ou de produire une "théorie " expliquant la signification des similitudes et des différences entre les «cas individuels ". En d'autres termes, quelles que soient les solutions apportées à des problèmes ponctuels, la comparaison classique reproduit toujours la "société ", comme le fait aussi, bien sûr, le concept de "théorie " lui-même, par opposition aux "données".

\section{Société $:$ Comparaison $=$ Socialité $:$ Analogie}

C'est de là que vient l'intérêt de Strathern pour l'analogie. Or, si l'on veut comprendre le mode d'analogie pratiqué par Strathern, il faut d'abord examiner comment elle substitue la « socialité » à la société.

Hormis lorsqu'elle parle du monde euro-américain, Strathern ne cherche généralement pas à créer des néologismes pour marquer des concepts (voir par exemple les relations mérographiques à la note 12 de ce texte). Aussi la socialité, comme beaucoup des termes qu'elle emploie, est-elle une expression familière détournée dans l'intention d'opérer une redescription: alors que la société est classiquement considérée comme une entité singulière et comme le "contexte" de l'interaction, la "socialité " évoque la manière dont les personnes, en Mélanésie, sont engendrées et entretenues dans leur vie quotidienne par une "matrice relationnelle» (Strathern 1996a: 53). Plus précisément, la socialité en Mélanésie compose les personnes; bien que celles-ci soient des entités singulières, elles sont en fin de compte "dividuelles " (plutôt qu' "individuelles»), dans le sens où elles sont "le site pluriel et composite des relations qui les ont produites" (Ibid.). Plus important encore, ce dividuel est androgyne et se déplace continuellement d'un état genré à un autre selon les circonstances sociales - sa différenciation interne est réprimée ou "effacée ", et l'aspect masculin ou féminin ressort au gré d'interactions ou d'échanges particuliers (le don est ainsi lui aussi genré au gré d'interactions spécifiques, puisque les Mélanésiens ne font pas de distinction entre sujet et objet). En bref, la personne n'est pas seulement «une» mais divisée - et les multiples relations sont des "fractions de l'un " (Strathern 2011a: 93). C'est pour cette raison qu'on ne voit pas les "hommes» contrôler les «femmes", même si l'imagerie du genre organise une bonne part de la vie mélanésienne. De même, si la personne est produite par des relations, cela ne signifie pas pour autant que la collectivité " fabrique » les personnes comme êtres sociaux, car cela impliquerait de réinstituer l'« échelle » verticale hiérarchique d'une société transcendante qui façonnerait l'individu. Strathern s'efforce de montrer, au contraire, que les personnes mélanésiennes sont perçues sur le mode de l'analogie : chacun est équivalent 
à un autre - de même échelle -; chacune contient les relations ou les échanges genrés qui l'ont constituée; chacun verra certaines des relations qui le composent réprimées ou coupées afin d'en faire apparaître d'autres ; chacune s'avère une reproduction-pas-tout-à-fait-exacte de l'autre (2004 [1991] : XX). En somme, le décentrement de la société et la découverte de la socialité mélanésienne indiquent que c'est l'analogie, et non pas la comparaison, qui se rapproche le mieux du mode mélanésien d'engagement avec l'autre.

\section{Écrire l'anthropologie, ignorer l'échelle}

Ce que fait Strathern à partir de cette découverte de la raison analogique est remarquable: elle l'intègre à l'écriture de ses données mélanésiennes et euro-américaines. Ce geste est encore plus radical qu'il n'y paraît: Strathern reconceptualise - et redécrit - l'entreprise anthropologique, un peu comme les premiers anthropologues du début du XXe siècle inventèrent un nouveau genre, la monographie, lorsqu'ils modifièrent leurs conceptions de la société et de la comparaison (Strathern 1987d). Or, Strathern développe son propre genre, contrairement aux premiers anthropologues qui faisaient appel à un certain modèle de la science, fondé en partie sur la description d'objets/sociétés individuels par des sujets/anthropologues. Nous pourrions appeler ce nouveau genre l' ethnographie analogique ", qui se calque sur la façon dont elle appréhende, et même incorpore, les pratiques mélanésiennes de savoir.

Prenant part aux débats de l'époque, Strathern développe ces idées dans Partial Connections (2004 [1991]). Les contributeurs à l'ouvrage Writing Culture (Clifford \& Marcus 1986 ; voir aussi Fabian 1983) avaient commencé à mettre en question les conventions modernes d'écriture ethnographique dans le cadre d'un dialogue avec Foucault. Ils estimaient que l'ethnographie était en proie à une "crise de la représentation", les anthropologues ne pouvant plus ignorer les relations de pouvoir et conserver l'autorité de "représenter " la vérité sur des Autres intemporels, qui sont l'objet de l'ethnographie. Aussi lançaient-ils un appel pour une ethnographie postmoderne, conçue comme fiction et écrite, sur un mode plus expérimental, à l'encontre des prétentions à l'objectivité, à la vérité et à la distinction anhistorique entre "eux" et "nous ". Même si, aux yeux de Strathern, nombre de ces arguments surgissaient "après coup " (Strathern 1987d) - les féministes s'étant mises à l'écriture expérimentale depuis un moment déjà -, cette volonté de reconceptualiser l'anthropologie et, par conséquent, l'écriture anthropologique entrait nettement en résonance avec sa propre tentative de redescription. Cependant, ces anthropologues proposaient aussi de remplacer l'anthropologue de terrain 
par des figures telles que le "voyageur " ou le " cosmopolite", propositions qui réinstituaient, chacune à leur manière, la "société » et l'" individu ", et donc la "comparaison» (2004 [1991] : 7-16). La réponse de Strathern fut de mettre plutôt en avant l'image du cyborg, qui rend bien la façon dont l'anthropologue est transformé par la pratique analogique de ses (relations avec ses) interlocuteurs mélanésiens. En outre, selon le portrait qu'en fait Strathern, le cyborg offre une réponse beaucoup plus complète que l'ethnographie postmoderne à la critique foucaldienne de l'"anthropologie " (Foucault [1969] la concevait généralement comme l'ensemble des discours visant à préserver la souveraineté du sujet humain - «L'Homme »).

Le cyborg a d'abord été popularisé par la chercheuse féministe Donna Haraway, dans un manifeste qui se voulait critique d'une certaine politique féministe à la recherche d'identités «naturelles» essentialistes, et qui plaidait pour un point de vue "partiel/partial» plutôt qu' " universel» (Haraway 1991 [1985]; voir aussi 1988). En écho à ces objectifs, sinon à ce langage, Strathern déploie la figure du cyborg pour mieux redécrire le projet de l'anthropologie (après tout, l'expression "connexions partielles" [partial connections] ne dit "ni plus ni moins que ce que dit, par exemple, la formule "écrire l'anthropologie" [writing anthropology]", 2004 [1991] : XXIX). Bien que le cyborg ne soit pas une figure mélanésienne, Strathern considère qu’il possède une capacité exceptionnelle à effectuer un travail social/analytique (presque) comme le ferait une personne mélanésienne : le cyborg ne « respecte aucune échelle, il n'est ni singulier ni pluriel, ni un ni plusieurs, mais un circuit de connexions qui relient des parties qui ne peuvent être comparées dans la mesure où elles ne sont pas isomorphes les unes aux autres" (Ibid.: 54, c'est moi qui souligne). Le cyborg est donc plus qu'une personne " individuelle» - plus que l'anthropologue - parce qu'il est l'anthropologue prolongé par son circuit de connexions, ce qui, dans le cas de Strathern, inclut des pratiques de savoir euro-américaines et mélanésiennes, ainsi qu'une technique d'écriture anthropologique. Autrement dit, dans l'écriture de Strathern, l'anthropologue n'écrit pas à propos des autres, dans la mesure où le cyborg ne réinstitue pas le point de vue des individus (ou de la société). Au contraire, toutes les autres positions et capacités convergent vers le circuit pour établir des « connexions sans hypothèse de comparabilité » (Ibid. : 38). L'étude de cette opération cyborgienne permet de mieux élucider pourquoi Strathern conserve une certaine positionnalité dans le texte - positionnalité à propos de laquelle on a pu écrire qu'elle se situait entre une description à la première personne et une autre à la troisième personne, et dont la perspective «individuelle» était absente 
(Viveiros de Castro \& Goldman 2009 et 2017 ; Holbraad \& Pedersen 2009 : en particulier 392, note 13).

En outre, Strathern propose une analogie visuelle afin d'aider les lecteurs à saisir l'écriture du cyborg: l'image de la poussière de Cantor, une image fractale dont la complexité (les relations internes) est autosimilaire à chaque échelle (c'est-à-dire le contraire de la société).

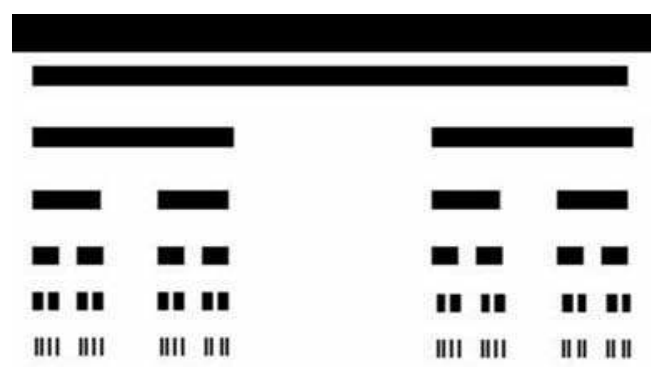

La poussière de Cantor

cf. James Geick, Chaos. Making a New Science, New York, Viking, 1987 (in Strathern 2004 : 3)

À la page 2 de la "Table des matières », Strathern présente cette fractale comme un "synopsis» de Partial Connections. Les chapitres du livre luimême sont bien sûr composés de différents niveaux de sections en paires, à partir desquels surgit un argument non linéaire. Or, comme le cyborg, l'image rappelle aussi la description que fait Strathern de la socialité et de la personne mélanésiennes : on considère que les personnes sont analogues dans la mesure où elles sont formées de paires de paires de relations - chacune d'elles comportant une "socialité" tout aussi complexe. Et pourtant, rappelons-nous qu'il faut pratiquer des coupes pour rendre visibles ou connaissables des ensembles spécifiques de relations et de personnes. On peut voir ces coupes et ces relations auto-similaires dans l'image ci-dessus. Ainsi Strathern communique-t-elle la façon dont elle produit son savoir: en établissant des analogies entre des ensembles de relations. Contrairement à l'argument de type linéaire-vertical que la pensée-société impose à l'écriture, le cyborg et la fractale - qui ne sont jamais qu'une reproduction-pas-tout-à-fait-exacte de la personne mélanésienne - permettent de penser et d'écrire une autre forme d'anthropologie.

Strathern suit ce modèle d'écriture dans Partial Connections et au-delà : elle juxtapose des vignettes - des récits de relations - mélanésiennes à des vignettes euro-américaines. L'objectif n'est pas de comparer, mais d'élucider l'un par l'autre, sans chercher à "abstraire» ou à produire une vision hiérarchique ou à échelles multiples. Strathern semble offrir à ses lecteurs l'expérience de sa compréhension ethnographique - voire 
l'expérience de la pratique ethnographique -, plutôt qu'un savoir ou une théorie sur des sociétés ou des cultures. En effet, elle montre à la fois par la forme et le contenu ce que c'est que d'essayer de voir, d'écrire et de comprendre le monde comme le font les Mélanésiens, avec tout le court-circuitage qu'implique évidemment la création de telles connexions.

Il est vrai que le cyborg et la poussière de Cantor ne réapparaissent pas dans les ouvrages postérieurs de Strathern, ce qui explique peut-être pourquoi on trouve peu de commentaires sur le rôle qu'ils jouent dans son approche de l'écriture et de la "comparaison" (Holbraad \& Pedersen 2009; Hirsch 2014). Or, cela ne signifie pas pour autant qu'ils ne sont pas pertinents pour comprendre la pratique actuelle de Strathern ; comme elle le laisse elle-même entendre, des discussions différentes peuvent masquer les origines de certaines idées (2004 [1991] : 54). Cela indique plutôt que certaines des idées ou des outils analytiques de Strathern ont pu cesser d'être saillants ou manifestes. Ainsi, en dépit de sa critique de la comparaison, il lui arrive encore, à l'occasion, d'utiliser le terme de façon moins critique (Strathern 1996b, 2003b, 2014a). Pour autant, elle n'a pas abandonné son projet d'élargir la comparaison. D'une part, le terme est parfois pertinent, surtout lorsqu'il s'agit pour Strathern de discuter ou de critiquer les "théories" qui effectuent des abstractions, plus ou moins poussées, à partir de leurs données (par exemple, la théorie de l'acteurréseau et la notion d'infinitude [Strathern 1996b] ; ou encore les anthropologues qui décrivent des relations en restant pris dans les conventions euro-américaines [Strathern 2014a: 44]). D'autre part, le travail critique et descriptif qu'elle a réalisé pour démontrer le pouvoir de l'analogie semble avoir été suffisant: la "comparaison" aurait été élargie avec succès et ne constituerait plus un "problème». En effet, comme elle l'explique dans un entretien à paraître avec Eduardo Viveiros de Castro et Carlos Fausto :

«[...] lorsqu'elle fonctionne bien, la déconstruction est en mouvement; c'est-à-dire qu'elle ne reste pas en place. C'est un processus temporel : vous ouvrez des choses et puis elles se referment, et vous les ouvrez, et elles se referment encore, et ainsi de suite. Je ne suis donc pas du tout gênée par le fait de m’être débarrassée du concept dans un contexte et de l'avoir utilisé dans un autre " (Strathern 2017).

Et bien sûr, Strathern continue d'attirer les gens dans son œuvre en les invitant à réfléchir aux problèmes de la description plutôt qu'en ignorant leur intérêt pour la comparaison. Dans sa préface à un ouvrage collectif, où elle est brièvement citée comme une théoricienne majeure de la comparaison, elle écrit avec humour :

«La comparaison est le jeu [de l'anthropologie] dans le sens à la fois le plus sérieux et le plus ludique du terme - on ne doit pas le dévoiler, mais y jouer. Et avec qui joue-t-on si ce n'est avec tel ou tel côté ?» (Strathern 2002 : XVII). 
Bien qu'elle ne précise pas ici en quoi ce jeu consiste, elle explique clairement comment s'y prendre pour y jouer : "commencez par les problèmes"(Strathern 2002: XVII). En prenant les problèmes de la comparaison très au sérieux, Strathern en est venue à considérer que le meilleur moyen d'élargir la comparaison, mais aussi l'anthropologie elle-même, est l'analogie - une relation moins hantée que la comparaison par la métaphysique de la société. Avec l'analogie, Strathern a en effet appris que les seules unités universellement " comparables » sont les relations.

\section{Relations}

Si décentrer la société et élargir la comparaison créent les conditions de base pour la redescription, cette dernière est mise en ouvre par l'étude analogique des relations, ou, tout simplement, l'étude des relations entre elles. Or, pour Strathern, les relations - c'est-à-dire les connexions et les distinctions conceptuelles et interpersonnelles qui nourrissent la vie sociale ne représentent pas seulement l'objet de la description anthropologique. Elles sont aussi ce qui nourrit l'anthropologie. En d'autres termes, les relations, dont le principe premier est qu'elles sont libres des contraintes d'échelle (2005b : 63), opèrent partout; elles sont à la fois la « relation de l'anthropologie» ou son outil, ainsi qu'un « outil, tout court, pour la vie en société » (Ibid. : 7). Si cela ressemble à un universalisme, c'est que c'en est probablement un. Les relations sont sans doute la seule donnée universelle que Strathern reconnaisse, même si la relation a sa propre contingence culturelle, qui est née du ferment social et intellectuel que fut la révolution scientifique, comme nous le verrons plus loin. Dans ce qui suit, un aperçu de la vision relationnelle de Strathern complétera mon analyse du versant "académique» de sa redescription. Nous pourrons ensuite nous tourner vers son versant "politique».

Il est à noter que le premier exposé explicite de Strathern sur la relation (The Relation, 1995) venait désamorcer une critique plus ancienne de sa vision relationnelle de l'anthropologie. Quelques années auparavant, James Weiner $(1993,2001)$ avait soutenu que mettre la relation au fondement de l'anthropologie était trop totalisant et empêchait de voir au-delà (Ibid.). Des critiques similaires ont été formulées par la suite, même si toutes ne proposaient pas (comme le faisait Weiner) de promouvoir l'étude de l'«être" ou l'"ontologie" comme alternative aux relations. Strathern évoque brièvement ces critiques dans la préface de Kinship, Law and the Unexpected (2005b), signifiant simplement son accord avec plusieurs de leurs aspects. Elle affirme cependant: 
«C'est avec mon propre conservatisme que je peux servir au mieux le nouveau radicalisme, conservant ainsi ce qui deviendra alors une position originale plutôt que d'en consommer de nouvelles! Je cherche donc à rester fidèle à un point de vue, non pas parce que je le défends, mais parce qu'il est plus fructueux de préciser - surtout à ce moment critique - ce qu'il a de si intéressant et qu'il deviendrait donc essentiel d'abandonner" (Ibid. : x).

Bien que Strathern évite ici de façon typique d'aborder frontalement la critique, on peut néanmoins lire Kinship, Law and the Unexpected, mais aussi le travail sur la relation qu'elle a effectué avant et depuis lors, comme offrant des réponses solides aux questions sur la centralité des relations dans l'analyse anthropologique. Elle démontre en effet que, quand bien même les anthropologues souhaiteraient aller au-delà des relations, l'anthropologie n'a sans doute pas encore les moyens de le faire.

L'héritage que nous a légué la révolution scientifique explique pourquoi nous sommes " coincés" avec les relations, pour ainsi dire (du moins pour l'instant). Strathern (1995, 2005b, 2014b) montre comment le concept de la relation a migré de la sphère scientifique à celle de la parenté, consolidant ainsi le lien entre production du savoir et relations sociales. Avant le XVII ${ }^{e}$ siècle, le mot "relation » était utilisé pour désigner le champ des relations logiques. Cependant, avec l'essor de la révolution scientifique, qui inclut de nouvelles formes d'association incarnées dans des "sociétés" (scientifiques et autres) alors en pleine expansion, les "relations" en vinrent à décrire des personnes dans la sphère de la parenté, et même au-delà. Il est difficile de déterminer pourquoi il en fut ainsi, mais souligner cette évolution - et notre usage continu des "relations " pour désigner à la fois l'activité conceptuelle et l'activité interpersonnelle - fait porter notre attention sur l'époque particulière dont les anthropologues sont encore partie prenante. En fin de compte, en mettant en lumière les origines de la vision relationnelle en anthropologie, Strathern nous révèle que les relations conceptuelles et interpersonnelles participent de la production du savoir lui-même, et que l'anthropologie n'est toujours pas capable d'échapper à cette contrainte. On ne peut savoir quelque chose qu'à travers cette vision relationnelle.

Cela ne veut pas dire que la science et l'anthropologie s'appuient sur les mêmes relations pour produire leurs savoirs. Au contraire, Strathern oppose la "relation anthropologique " à la "relation scientifique ". Cette dernière met en ouvre une dyade différente, partageant le savoir entre relations "inventées» et relations "découvertes». Ces différences mises à part, la relationnalité de l'anthropologie s'est développée sous l'influence implicite et explicite de la science. Implicitement, l'anthropologie et les sciences sociales en général ont excellé à la "découverte " de relations qui 
sont déjà là, plutôt qu’à l'« invention » de nouvelles relations (2005b : 39). En revanche, une influence explicite de la science sur l'anthropologie a été l'idée même que l'on pouvait comprendre la société par le biais de certains protocoles et méthodes. L'une des méthodes proposées était la statistique, dans laquelle des données étaient recueillies quantitativement pour trouver des corrélations. Sous ce régime, les données sont " comprises comme des éléments individuels au même titre que les personnes peuvent être considérées comme des individus et la société comme les connexions entre eux» (Ibid. : 38). C'est ainsi, remarque Strathern, que l'anthropologie s'est retrouvée aux prises avec plusieurs des problèmes analytiques associés à la pensée-société dont nous avons discuté précédemment(voir les deux premières parties). En effet, le point de vue de la science, ce que Haraway (1988) appelle le God trick (l'« entourloupe de Dieu»), est aussi le point de vue de la société.

En dépit du rôle essentiel qu'a joué la science dans le développement de l'anthropologie, Strathern ne prétend pas que la nature "duelle " de la relation anthropologique (sa mobilisation des relations conceptuelles et interpersonnelles) soit le simple produit de la révolution scientifique. Au contraire, le recours à la fois aux relations conceptuelles et aux relations interpersonnelles est quelque chose que l'on retrouve chez toutes les populations du monde. Néanmoins, en ce qui concerne l'anthropologie, la connexion culturelle et historique entre la relation anthropologique et la production du savoir revêt une importance particulière. Sa provenance euro-américaine ne signifie pas pour autant qu'elle n'ait pas de portée générale; Strathern a clairement démontré que nos analyses, lesquelles sont nécessairement contingentes, peuvent encore nous aider à mieux voir les "autres" et à nous voir "nous-mêmes", mais aussi à atténuer les différences entre «eux» et "nous» - dès lors que nous nous y efforçons. C'est même plutôt la relation entre la Relation et le savoir qui signale son utilité pour l'anthropologie. Car dans sa volonté « disciplinaire » de penser relationnellement, de se déplacer entre le conceptuel et l'interpersonnel pour produire du savoir sur la vie sociale, «l'anthropologie en arrive à une certaine vérité de la socialité qui ne peut être saisie d'aucune autre façon " (2005b: 8). En d'autres termes, sans une vision relationnelle pour produire nos descriptions, nous n'en saurions véritablement pas autant sur la vie sociale. On pourrait même dire que, sans la relation pour nous permettre d'acquérir de nouvelles perspectives, nous ne pourrions pas apprendre et redécrire perpétuellement ce que nous pensons savoir.

Enfin, la vision relationnelle de Strathern doit être distinguée du tournant ontologique actuel (Viveiros de Castro 1998, 2011 ; Henare, Holbraad \& Wastell 2007 ; Holbraad, Pedersen \& Viveiros de Castro 
2014 ; Holbraad \& Pedersen 2014, 2016) avec lequel elle est (au moment où j'écris ces lignes) souvent confondue - la résonance de ce tournant avec la critique de Weiner semblant avoir été éclipsée. Non pas que Strathern ignore que certaines pratiques soient ontologiques (par exemple certaines pratiques rituelles chez les Mélanésiens, ou encore le droit), dans la mesure où elles constituent les phénomènes en question plutôt qu'elles ne les "représentent» (comme le ferait une pratique épistémologique). On ne peut pas dire non plus qu'elle ne s'intéresse pas aux discussions menées par les chercheurs, et en particulier les anthropologues, que l'on identifie le plus à ce tournant. Bien au contraire, ceux-ci se méfient comme elle des concepts euro-américains capables d'occulter les «mondes" de ceux à propos desquels ils ou elles écrivent, et c'est en définitive bel et bien pour cette raison que Strathern commente leurs travaux ${ }^{17}$. Néanmoins, Strathern considère la relation anthropologique comme un artefact épistémologique. Elle suggère également que la recherche d'un autre moyen de fonder le projet anthropologique revient à ignorer l'inévitable :

«Malgré tout ce que [la relation] nous permet de savoir sur les relations non épistémiques, sa limite est (évidemment) la forme qu'elle prend [soit une dyade composée du conceptuel et de l'interpersonnel]. Car quand bien même [la relation] est utile pour élucider l'autre côté des choses, particulièrement dans le cas des sociétés situées hors de l'orbite de celles des Lumières et de la révolution scientifique, les choses demeurent en effet "autres", c'est-à-dire qu'elles sont toujours vues en relation au point de vue du moment. C'est bien là l'artifice du "monde unique" euro-américain, et une surprise ultime qui ne devrait pas surprendre. Ce qui advient à la relation duelle quand les anthropologues découvrent qu'ils peuvent compter des mondes de différentes manières est, précisément, rien. Bref, la relation ne va pas disparaître» (2005b : 91, c'est moi qui souligne).

Il convient de rappeler que le tournant ontologique se veut en partie la critique de l'épistémologie comme théorie euro-américaine de la connaissance, selon laquelle il y a "un monde" et plusieurs cultures (ou constructions) du monde. Ce tournant propose de déplacer l'épistémologie,

17. Bien que le "tournant ontologique" soit composé d'un groupe de chercheurs venus de différents horizons, Strathern s'intéresse principalement aux anthropologues qui s'efforcent de développer le concept d'ontologie. L'ontologie a certes été pensée de multiples manières au sein de ce groupe en plein essor, mais cela n'est pas pertinent pour comprendre la réponse qu'y oppose Strathern. En effet, celle-ci s'est clairement exprimée sur les effets que peut avoir le fait d'accorder la priorité au développement de concepts en général, et à l'ontologie en particulier, et ce, quelle qu'en soit la définition. Or, à ces critiques nous pouvons en ajouter une autre qui peut être déduite de son œuvre plus vaste: l'ontologie privilégie un concept philosophique éminemment euroaméricain (et plutôt unitaire d'ailleurs, puisqu'il évoque la société, l'individu et la comparaison) et lui assigne la tâche de description ethnographique. Bien que Strathern soit consciente du fait que les chercheurs ne peuvent complètement échapper à leur langage, le recours à des concepts aussi chargés, et en particulier à des concepts qui peuvent évoquer la pensée-société, est précisément ce qu'elle a cherché à éviter dans sa définition de la contribution spécifique de l'anthropologie. 
afin de pouvoir découvrir "plusieurs mondes " ou ontologies (par exemple, dans le cas des mondes amazoniens décrits par Viveiros de Castro [1998], il y a une culture et plusieurs natures). Ce que Strathern désigne quand elle parle de "surprise ultime qui ne devrait pas surprendre", c'est le fait que même lorsque nous découvrons des mondes hors de «l'orbite de ceux des Lumières" ceux-ci sont, en fin de compte, toujours vus en relation à un autre monde. En d'autres termes, même lorsque nous découvrons des mondes différents, nous ne pouvons échapper à la relation qui existe entre eux et donc à l'épistémologie.

Strathern continue de renforcer ce point dans ses derniers écrits. Ainsi, face aux tentatives pour développer une anthropologie «au-delà même de l'humain" - et donc des relations perçues comme étant strictement sociales -, Strathern observe que les relations permettent encore partout de penser. Par exemple, en réponse à l'appel de Haraway (2003) pour une étude des relations entre l'humain et l'animal, Strathern se recentre avec humour sur la relation elle-même, qu'elle nomme notre "concept compagnon " (2014b : 8). Ce n'est pas tant à la relation comme " concept " que Strathern s'intéresse qu'au travail relationnel cohérent qu'elle effectue entre l'humain et le non-humain - tant il est vrai que les relations nous «traquent", peu importe où nous sommes et ce que nous sommes.

En outre, Strathern insiste de plus en plus sur les conséquences de privilégier les différences entre les mondes plutôt que de s'intéresser aux relations. Les anthropologues devraient, selon notre auteure, considérer l'importance des relations pour l'être lui-même. Sinon, ils pourraient perdre leur capacité à percevoir les transformations - y compris la façon dont les relations peuvent transformer ceux qui vivent selon un mode d'être ontologique en ces "autres" qui adhèrent à un mode de savoir épistémologique -, et ce, que nous le voulions ou non (voir par exemple 2005b : 145-146, 2014c). Strathern a ainsi donné le nom d' «intervention » à la prise en compte, par les anthropologues, des conséquences sociales des relations (2014c: 35); elle est d'ailleurs consciente du besoin qu'elle a d'intervenir sur cette question (sans doute réécrit-elle aussi l'analyse foucaldienne des "procédures d'intervention »- qui créent les conditions de la formation de concepts - tout en postulant que la relation est toujours déjà une intervention, avant et au-delà du « concept $»)^{18}$.

18. Il convient de rappeler que, pour Foucault, les "procédures d'intervention " sont l'un des trois moyens par lesquels se forment les concepts au sein de formations discursives spécifiques (1969: 79). Ces procédures peuvent être de types variés, y compris par exemple les «techniques de réécriture " (Ibid.), ce qui renvoie à la redescription formulée par Strathern. Or, Foucault observe aussi qu'un système de formation conceptuelle est défini par les relations qui le constituent, et, en particulier, par la «manière par exemple dont l'ordonnance des descriptions ou des .../... 
$\mathrm{Vu}$ sous cet angle, l'invitation que lance Strathern à ceux et celles qui s'efforcent de développer le concept d'ontologie prend toute sa dimension critique :

«Peut-on imaginer un univers de chercheurs où cela [élaborer des concepts] ne serait pas un devoir primordial ? [...] [L]e lieu de la vérité se trouve peut-être ailleurs que dans les concepts. Ce qui voudrait dire que les préoccupations morales se situent elles aussi ailleurs" (2012: 403).

Bien sûr, Strathern ne nie pas que le mode d'argumentation et l'utilisation des termes - ce que l'on pourrait communément nommer le travail conceptuel - puissent être des préoccupations pour ces "ailleurs" réels ou imaginaires. Néanmoins, elle exhorte les anthropologues à penser aux relations nées de leurs différentes rencontres - en particulier à la manière dont ils entrent en relation avec ces autres modes de description, et dont ils peuvent laisser ceux-ci transformer leurs propres modes de description, voire même transformer leurs concepts anthropologiques des concepts. En d'autres termes, les relations ne sont pas seulement "pensées", elles sont continuellement mises en œuvre et ont une valeur à la fois vitale et morale : elles sont au cœur de la façon dont les personnes sont diversement régies par «elles-mêmes» et par "d'autres ». En bref, les relations, au-delà des concepts eux-mêmes, ont des conséquences pour la "politique " de l'anthropologie.

\section{Une politique de la redescription}

Strathern n'est pas connue pour débattre ouvertement de "politique", anthropologique ou autre. On devrait pouvoir facilement deviner pourquoi, à présent. La "politique», tout comme la société, est une notion euro-américaine fort chargée et que l'on inscrit trop aisément dans nos ethnographies. En outre, si elle n'est pas manipulée avec précaution, elle peut occulter plutôt qu'élucider les relations qui y ont cours. La "politique ", qui évoque la lutte autour des modes de pouvoir, et surtout de gouvernance, pèse déjà de tout son poids. En effet, même si de nombreux chercheurs ont critiqué le concept de société, l'État, et toutes

[Suite de la note 18] récits est liée [reliée] aux techniques de réécriture" (Ibid. : 80). Aussi la relation chez Foucault est-elle plus qu'un concept, elle est également ce qui rend possible les concepts, lesquels surgissent entre ordonnance et réécriture. (Par ailleurs, selon Dreyfus et Rabinow [1982: 63], on méconnaît souvent cette préoccupation centrale de Foucault pour la façon dont les relations constituent le discours.) Chez Strathern aussi les relations précèdent les concepts, même si, de ce fait, la relation est ce sur quoi elle porte son attention. Ses modes de réécriture et de redescription élargissent le champ de l'anthropologie du simple concept ou discours, aux conséquences sociales des relations conceptuelles et interpersonnelles : telle est son intervention. 
sortes de "totalités ", ils n'ont pas pour autant abandonné la politique; et l'attention portée à cette dernière reste en grande partie la mesure d'une réflexion intellectuelle (critique) correcte. Or, comme dirait Strathern, pareille insistance sur la politique, au lieu de transformer son fondement, ne fait que le reproduire : à savoir la société elle-même, dont la politique fait partie intégrante. Mais cela ne veut pas dire qu'on ne retrouve pas dans l'anthropologie de Strathern une forme de politique. D'abord, l'entreprise de redescription qu'elle propose vise à élargir non seulement notre vision de la société, de la comparaison et de la théorie, par exemple, mais aussi ce que l'on peut considérer comme la critique - et donc comme la politique - anthropologique. Ensuite, et surtout, toute l'œuvre de Strathern depuis The Gender of the Gift conteste les perspectives sous-jacentes aux modes de gouvernance - qui représentent, après tout, une sphère du politique façonnée par nos concepts. En effet, elle montre dans toute son œuvre comment l'anthropologie peut contribuer à la politique : par une stratégie de redescription (ethnographique), régulière et à long terme, qui permet de démontrer comment les relations constituent la vie humaine de manière très diverse. Il faut par conséquent s'intéresser à la façon dont notre savoir est conditionné par des arrangements relationnels spécifiques.

Commençons par la refiguration de la critique effectuée par Strathern, puisque c'est là le seuil anthropologique qui ouvre sur la politique au sein de l'université. Si je me suis penchée précédemment sur l'exégèse critique du couple "société/individu" au sein des approches anthropologiques dominantes, je dois ajouter ici que cette exégèse constitue aussi une critique de la critique anthropologique - en particulier en ce qui concerne ses formes courantes (les modes d'argumentation) et ses objets. Premièrement, les formes classiques de la critique anthropologique reflètent la métaphysique de la société : comme avec la comparaison, on oppose des données à une théorie globale afin de démontrer sa vérité ou sa fausseté. La redescription - décentrer des concepts et rechercher des relations analogiques - produit un type d'argument plus horizontal, qui ne vient pas abstraire une vérité théorique transcendante, puisqu'il est toujours immanent au récit analogique de Strathern, lequel circule à travers ses propres ethnographies et celles des autres. En effet, tel qu'on l'a vu au départ, dans la recherche la critique consiste à « multiplier et diviser à nouveau les résultats de toute thèse particulière " (Strathern 2006 : 199). Deuxièmement, son approche de la critique interroge de manière détournée le fait que l'«anthropologie critique» se reconnaît d'abord et avant tout par ses objets. Il convient ici de souligner que l'objectif de Strathern n'est pas de minimiser les formes critiques et les préoccupations politiques courantes. Non seulement elle s'y intéresse, mais il serait 
contraire à sa définition même de la critique d'écarter directement d'autres points de vue. Cependant, son approche de la critique contraste énormément avec une certaine " anthropologie critique » qui puise son inspiration au cœur des traditions marxistes et foucaldiennes (aussi différentes soientelles l'une de l'autre) et consiste à dénoncer les usages et les abus du pouvoir au cours du temps, et elle est souvent considérée comme étant beaucoup moins politique. En d'autres termes, il est entendu que l'anthropologie critique doit avoir pour objet l'histoire des exclusions politiques et économiques, des processus disciplinaires, des luttes et des violences.

Strathern met en question ce que l'on entend par anthropologie critique: plutôt que de décrire directement des luttes de pouvoir ponctuelles, ses arguments expriment une stratégie politique à long terme pour une discipline qui occupe une place particulière au sein des "sciences". Si chaque science a pour tâche d'informer sur les enjeux de son domaine d'expertise, le rôle de l'anthropologie est de produire du savoir sur les relations. Et l'anthropologie est bien placée pour révéler que les relations ne fonctionnent pas toujours comme les détenteurs du pouvoir le voudraient.

Strathern a proposé un point de vue essentiel sur les relations à travers un éventail impressionnant de thèmes. Mais si l'on veut comprendre la dimension politique de l'intérêt qu'elle porte aux relations, il faut tout reprendre depuis le début. Rappelons d'abord qu'elle a effectué sa redescription de la "société" mélanésienne dans le contexte de la transformation néolibérale impulsée par Thatcher (et Reagan). La fameuse déclaration de Thatcher, que je peux maintenant citer en entier, exprimait bien sûr cette transformation : "Il n'y a pas de société. Il y a des hommes et des femmes et il y a des familles" (cité in Strathern 1996a: 53). Pour Thatcher, supprimer la notion "abstraite» de société permettait de concrétiser l'«individu» et donc l'individualisme - l'idée que les personnes sont autonomes, possessives, maximisatrices. D’un point de vue politique, cela justifiait les attaques contre les programmes de sécurité sociale :

"Voyez ce qui est arrivé. D’un seul coup, le thatchérisme a pu rassembler toutes sortes de collectivités et d'organisations qui faisaient du social et s'en débarrasser. Leur caractère social ne leur donne plus de légitimité parce que la société n'existe plus » (Ibid. : 54).

En fin de compte, observe Strathern, "là où l'individu est produit "par opposition" à la société, une telle manœuvre dissimule les formations sociales et les relations de pouvoir" (Ibid.). Il ne faut pas oublier que la révolution conservatrice est toujours en cours. Ni négliger que, si on 
" regarde la parenté anglaise, on peut trouver des idées et des questions qui confortent les idées de Thatcher", ainsi qu'elle l'évoque au sujet d'After Nature (1992a) dans un entretien à paraître. Par conséquent, l'appel de Strathern pour une anthropologie qui ne tienne pas la "société " et l'« individu » pour acquis, et qui mette en avant l'importance des relations, non seulement favorise une meilleure description, mais offre à la discipline une vision politique à long terme. De fait, il s'agit de transformer la pensée-société plurielle - une pensée qui est déjà en train de changer, de devenir "post-plurielle ", et ce, pas seulement en raison du croisement entre les bouleversements technologiques (reproduction et génétique) et les effets du néolibéralisme (tel que nous le rappelle Sarah Green [2017: 5]). Le pluralisme change aussi parce que Strathern elle-même en a "rendu la pluralité explicite» (1992a: 7), et l'a décentrée en tant que mode de description. Et pourtant, il est évident que pour Strathern, aller au-delà du pluralisme demandera encore plus d' "ingéniosité" (Ibid.: 4) - de l'écriture et de l'écriture encore - et pas seulement de sa propre plume. La manière dont nous tous allons redécrire les choses est importante.

J'ai souligné que l'objectif de Strathern était de produire de meilleures descriptions de la Mélanésie, mais aussi du monde euro-américain - et pas seulement en tant que formations sociales foncièrement opposées, comme certains critiques ont pu l'affirmer. En effet, la redescription du monde euro-américain par le biais d'une anthropologie mélanésienne et cyborgienne permet à Strathern de voir des continuités à travers les différences, et d'étudier la manière dont les concepts posent des défis politiques et juridiques. Par exemple, dans son étude des innovations apportées par la procréation médicalement assistée, elle rend explicite les continuités au sein de la différence et du changement:

"La biotechnologie a introduit dans le domaine de la gestion du corps le type de séparations, de coupures et de combinaisons qui a toujours caractérisé les relations entre les personnes" (2005b : 30).

Si ces coupures rappellent les manières mélanésiennes d'entrer en relation, c'est le langage qui distingue les Euro-Américains :

"le fait est que les Euro-Américains ne parlent pas toujours des relations de façon très claire [...]. [Et] l'une des raisons de la pénurie d'idiomes relationnels est la surdétermination des autres idiomes" (Ibid. : 30-31).

Cette surdétermination des idiomes rend difficile, par exemple, de penser et de dire que la mère et le foetus sont à la fois distincts et imbriqués. Dans la lutte engagée pour déterminer la priorité des droits, les débats font rage quant à savoir si le foetus est indépendant ou non de la mère. 
Strathern remarque que mère et fotus doivent être séparables pour qu'une relation puisse s'établir entre eux, ce qui ne signifie pas qu'ils ne font pas partie l'un de l'autre. Cette perspective semble néanmoins hors de portée, et les débats continuent. Une relation ne sera jamais un sujet de droits (comme l'est l'individu) dans la loi euro-américaine. Cependant, l'argument plus général de Strathern est qu'au sein d'une socialité qui est universelle, les différentes configurations de relations sociales et de relations conceptuelles ont des effets différents. Et certains de ces effets concourent, intentionnellement ou non, à façonner la politique et le savoir.

C'est pourquoi Strathern suggère aux anthropologues d'être attentifs non seulement aux configurations passées des relations, mais aussi à leurs réarrangements actuels. Les formes contemporaines d'expression, même lorsqu'elles semblent inoffensives, peuvent mettre en route des programmes dangereux. L'analyse que fait Strathern de l'audit en est un bon exemple (Strathern 2000a). Alors que l'audit semble incarner des valeurs que les universitaires devraient pouvoir défendre, telles que la responsabilité et la transparence, il affecte notre manière de savoir et peut-être même ce que nous savons, lorsqu'il est appliqué - par exemple dans le cadre de l'enseignement supérieur. La prolifération actuelle des rituels de vérification des «bonnes pratiques» et de l'«efficacité économique» commence à menacer l'enquête ouverte qu'ils prétendent promouvoir. Cela a des conséquences particulières pour l'anthropologie, et surtout pour l'ethnographie, qui recueille des «données" sans connaître toutes leurs applications immédiates.

Comment les anthropologues peuvent-ils répondre au danger qui menace l'enquête ouverte? (Car ils devront bien le faire, sinon la "réponse" sera imaginée pour eux.) Strathern propose une approche "politique » qui prenne position vis-à-vis des politiques de gouvernement (Strathern 2000a : 289-291) : les anthropologues doivent non seulement reconnaitre les termes par lesquels les rationalités gouvernementales se propagent, mais également être en mesure de préciser le rôle spécifique de l'ethnographie. Strathern nous met pourtant en garde: produire de nouvelles ethnographies des politiques publiques - ou en produire de meilleures - n'est pas une réponse en soi. Les anthropologues devraient plutôt chercher à se démarquer du langage bureaucratique qui tente de les englober - la bureaucratie ayant, après tout, une capacité singulière à absorber les savoirs neufs et exogènes pour les détourner à ses propres fins.

Si la contribution critique et politique de Strathern semble s'appliquer principalement aux pratiques de savoir euro-américaines, c'est qu'elle l'a voulu ainsi. Son rôle, dit-elle, est de critiquer ses propres pratiques de savoir plutôt que celles des autres (Strathern 2017). Et pourtant, 
les pratiques de savoir euro-américaines ont une portée absolument démesurée. Il est essentiel d'y prêter attention parce qu'elles peuvent affecter la façon dont les autres sociétés s'organisent et se conçoivent ellesmêmes sur le plan politique. Strathern insiste là-dessus dans un article récent, où elle revisite un rapport rédigé dans les années 1970 qu'elle aurait aimé avoir composé autrement. En particulier, elle aurait aimé l'avoir écrit sans le concept d' "ethnicité", qu'elle avait utilisé à l'époque pour décrire les différents groupes ayant migré vers Port Moresby, la capitale de la Papouasie-Nouvelle-Guinée. Sa méfiance vis-à-vis de l'ethnicité est due à la façon dont les descriptions scientifiques des conflits «ethniques » en Papouasie-Nouvelle-Guinée convergent avec les descriptions politiques et gestionnaires. Il n'est pas sans conséquence, dit-elle, d'affirmer qu'il y a «toujours" eu des «conflits ethniques » en PapouasieNouvelle-Guinée, ce qui suppose un conflit fondé sur les différences et les similitudes perçues entre les groupes. En effet, le concept de conflit ethnique en est peut-être venu à façonner la pratique, voire à la motiver. Dans cette optique, Strathern redécrit son ancien rapport oublié; elle décentre le terme "ethnique » qu'elle avait alors employé pour décrire les différents groupes, et suggère que ses interlocuteurs de l'époque se voyaient les uns les autres au moyen d'une analogie morale, en étant conscients de la socialité qui les constituait tous. En d'autres termes, "il n'y avait aucun terrain commun ou aucune perspective commune qui seraient extérieurs aux entités que l'on avait réunies, de la manière dont on peut considérer que les références euro-américaines à l'humanité ou à la citoyenneté leur sont extérieures" ([Strathern 2011a: 96, c'est moi qui souligne] elle aurait pu placer "société" aux côtés de "citoyenneté" et "humanité»). Aujourd'hui, bien sûr, les choses ont sans doute changé pour les migrants de Moresby; il se peut même qu'ils se voient en termes ethniques. Mais la question demeure: comment les conflits en Papouasie-Nouvelle-Guinée en sont-ils venus à ressembler à des conflits "ethniques"? Il peut certes y avoir plus d'une réponse à cette question, mais selon Strathern, ces descriptions tenaces de "conflits ethniques", produites par les anthropologues et les entités gouvernementales ou non gouvernementales qui s'en sont inévitablement inspirées, ont joué un rôle important dans cette histoire.

"Si ce n'est la politique identitaire [ethnique], alors quelle politique?", demande Strathern (Ibid.: 126), revenant sur un commentaire de Pedersen (2011). Bien qu'elle attribue à Pedersen la réponse, c'est bien de sa propre réponse qu'il s'agit; et ce, depuis fort longtemps. La politique de Strathern consiste en définitive à révéler "l'inaliénabilité des relations entre les personnes, et donc leur enchevêtrement » (Strathern 2011a: 126). 
Cela ne veut pas dire, et j’insiste là-dessus, qu'elle envisage les relations comme étant intrinsèquement "bonnes" (elles peuvent être source de division et de violence, après tout). Les relations sont, tout simplement, une réalité permanente, et les anthropologues doivent faire en sorte que cette réalité soit pleinement reconnue, à l'opposé des politiques individualisantes. De même, pour Strathern, l'enchevêtrement réciproque des personnes ne signifie pas que nous sommes tous fondamentalement les mêmes, ni d'ailleurs tous fondamentalement différents. Il y a de l'unité dans la diversité. Strathern concède qu'il s'agit là d'une idée un peu euro-américaine, "mais ce n'est pas bien grave. Dans plusieurs contextes ce serait une bonne chose, et j'y ai moi-même fait appel en retournant constamment aux données mélanésiennes" (Ibid.: 124). Or, retourner maintes et maintes fois aux données mélanésiennes ne signifie pas simplement les opposer au monde euro-américain afin de mieux comprendre celui-ci (comme certains commentateurs ont pu l'imaginer). Au contraire, l'écoute attentive de ce que les autres ont à dire - voir et écrire avec eux peut aussi nous permettre d'être encore plus "nous-mêmes". Ainsi, toute l'œuvre de Strathern se résume à un projet à la fois anthropologique et politique, à savoir aller au-delà de nos cadres analytiques surdéterminants afin d'écrire au plus près de la perspective des autres: la redécrire. Strathern présente cela comme une invitation "non seulement à s'imaginer savoir une chose à travers une autre, mais aussi à expérimenter ce que ce serait en pratique d'écrire à propos des migrants de Hagen, alors qu'on est en train d'écrire sur la Corse ou la Mongolie des Darhad " (Ibid.: 127). En d'autres termes, elle nous invite, non pas à reproduire son mode redescriptif, mais à pratiquer une anthropologie qui s'apparente à la sienne; une anthropologie qui réécrive perpétuellement ce que nous pensons savoir en mettant en œuvre la somme particulière des relations - les personnes, les lieux, les œuvres diverses... - que chacun d'entre nous rencontre. Est-ce que l'un d'entre nous pourrait, un jour, se comparer à elle? Peut-être pas tout à fait.

Traduit de l'anglais par Arianne Dorval

Wilfrid Laurier University Department of Religion and Culture, Waterloo (Canada) alebnera@wlu.ca

MOTS CLÉS/KEYWORDS: Marilyn Strathern - méthodologie/methodology - redescription relation - concepts - comparaison/comparison - société/society - individu/individual. 
Allard, Olivier

2014 «Introduction : Marilyn Strathern et l'anthropologie française ", Tracés hors-série 14 : Traduire et introduire: 167-173.

\section{Borič, Dušan}

2010 "Arriving at a Good Description : Interview with Professor Dame Marilyn Strathern ", Journal of Social Archaeology 10 (2) : 280-296.

Clifford, James \& George Marcus, eds 1986 Writing Culture. The Poetics and Politics of Ethnography: A School of American Research Advanced Seminar. Berkeley, University of California Press.

Corsín Jiménez, Alberto

2015 «The Capacity for Re-Description : Environments for Hyphens ", in Matei Candea et al., eds, Detachment. Essays on the Limits of Relational Thinking. Manchester, Manchester University Press : 179-196.

\section{Deleuze, Gilles \& Félix Guattari}

1991 Qu'est-ce que la philosophie?

Paris, Minuit («Critique»).

Dreyfus, Hubert L. \& Paul Rabinow

1982 Michel Foucault,

Beyond Structuralism and Hermeneutics.

Chicago, University of Chicago Press.

Edwards, Jeanette \& Maja Petrovič-Šteger

2011 «Introduction: On Recombinant Knowledge and Debts That Inspire", in Jeanette Edwards \& Maja Petrovič-Šteger, eds, Recasting Anthropological Knowledge. Inspiration and Social Science. Cambridge-New York, Cambridge University Press : 1-19.

Fabian, Johannes

1983 Time and the Other. How Anthropology Makes Its Object. New York, Columbia University Press.
Foucault, Michel

1969 L’Archéologie du savoir. Paris, Gallimard («Bibliothèque des sciences humaines »).

Gell, Alfred

1999 "Strathernograms, or, the Semiotics of Mixed Metaphors ", in The Art of Anthropology. Essays and Diagrams.

Ed. by Eric Hirsch. London-New Brunswick, Athlone Press : 29-75.

Gingrich, Andre \& Richard G. Fox

2002 Anthropology, by Comparison.

Foreword by Marylin Strathern.

London-New York, Routledge.

Green, Sarah

2017 «Conclusion. Thinking Through Proliferations of Geometries, Fractions and Parts ", in Ashley B. Lebner, ed., In Redescribing Relations: Strathernian Conversations on Ethnography, Knowledge and Politics. New York, Oxford, Berghahn Books.

\section{Greenhouse, Carol}

2017 "Scales of Justice", in Ashley B.

Lebner, ed., Redescribing Relations.

Strathernian Conversations on Ethnography,

Knowledge and Politics. New York,

Berghahn Books [à paraître].

\section{Haraway, Donna}

1988 "Situated Knowledges: The Science Question in Feminism and the Privilege of Partial Perspective ", Feminist Studies 14 (3) : 577-599.

1991 [1985] «A Cyborg Manifesto :

Science, Technology, and Socialist-

Feminism in the Late Twentieth Century ", in Simians, Cyborgs and Women.

The Reinvention of Nature. New York, Routledge : 149-181.

2003 The Companion Species Manifesto. Dogs, People, and Significant Otherness. Chicago, Prickly Paradigm Press. 
Henare, Amiria, Martin Holbraad

\& Sari Wastell

2007 Thinking through Things.

Theorising Artefacts Ethnographically.

London, Routledge.

Hirsch, Eric

2014 «Melanesian Ethnography and the Comparative Project of Anthropology :

Reflection on Strathern's Analogical

Approach ", Theory, Culture \& Society

$31(2-3)$ : 39-64.

Holbraad, Martin \& Morten Axel Pedersen

2009 «Planet M: The Intense Abstraction of Marilyn Strathern ", Anthropological

Theory 9 (4) : 371-394.

2016 The Ontological Turn.

An Anthropological Exposition.

Cambridge University Press [à paraître].

Holbraad, Martin, Morten Axel Pedersen

\& Eduardo Viveiros de Castro

2014 "The Politics of Ontology:

Anthropological Positions ", Theorizing the Contemporary, Cultural Anthropology [http ://culanth.org/fieldsights/462-the-politics-of-ontology-anthropological-positions]

Josephides, Lisette

1991 «Metaphors, Metathemes, and the Construction of Sociality:

A Critique of the New Melanesian

Ethnography ", Man 26 (1) : 145-161.

Josephides, Lisette, Nigel Rapport

\& Marilyn Strathern

2015 "Dialogue ", in Lisette Josephides, ed., Knowledge and Ethics in Anthropology.

Obligations and Requirements. London-

New York, Bloomsbury: 191-229.

Lebner, Ashley \& Sabine Deiringer

2008-2009 "Editors' Note",

Cambridge Anthropology 28 (3) : 1-5.

Needham, Rodney

1975 "Polythetic Classification:

Convergence and Consequences",

Man 10 (3) : 349-369.
Pedersen, Morten Axel

2011 "Non-Identity Politics",

Common Knowledge 17 (1) : 117-122.

Pitt-Rivers, Julian

1974 "The Kith and the Kin", in Jack Goody, ed., The Character of Kinship. Cambridge, Cambridge

University Press : 89-106.

Power, Michael

2000 The Audit Implosion. Regulating

Risk from the Inside. London, ICAEW.

Radcliffe-Brown, Alfred Reginald

1952 Structure and Function in Primitive

Society. With a Foreword by E. E. Evans-

Pritchard and Fred Eggan. London,

Cohen \& West.

Strathern, Marilyn

1972 Women in Between. Female Roles

in a Male World: Mount Hagen, New

Guinea. London-New York, Seminar Press.

1980 "No Nature, No Culture:

The Hagen Case ", in Carol P. MacCormack

\& Marilyn Strathern, eds, Nature, Culture

and Gender. Cambridge-New York,

Cambridge University Press : 174-222.

1981 "Culture in a Netbag:

The Manufacture of a Subdiscipline in Anthropology ", Man 16 (4) : 665-688.

1985 "Discovering "Social Control” ", Journal of Law and Society 12 (2) :

111-134.

1987a «An Awkward Relationship:

The Case of Feminism and Anthropology", Signs 12 (2) : 276-292.

1987b "Conclusion", in Marilyn

Strathern, ed., Dealing with Inequality.

Analysing Gender Relations in Melanesia

and Beyond. Cambridge-New York,

Cambridge University Press : 255-277.

1987c "Introduction ", in Marilyn

Strathern, ed., Dealing with Inequality.

Analysing Gender Relations in Melanesia

and Beyond. Cambridge-New York,

University of Cambridge Press : 1-33. 
1987d «Out of Context: The Persuasive Fictions of Anthropology [and Comments and Reply]", Current Anthropology 28 (3) : 251-281.

1988 The Gender of the Gift. Problems with Women and Problems with Society in Melanesia. Berkeley, University of California Press.

1992a After Nature. English Kinship in the Late Twentieth Century. CambridgeNew York, Cambridge University Press. 1992b « Parts and Wholes: Refiguring Relationships in a Postplural World", in Reproducing the Future. Anthropology, Kinship, and the New Reproductive Technologies. Manchester, Manchester University Press : 90-117.

1995 The Relation. Issues in Complexity and Scale. Cambridge, Prickly Pear Press.

1996a «Debate: The Concept of Society Is Theoretically Obsolete ", in Tim Ingold, ed., Key Debates in Anthropology. LondonNew York, Routledge : 50-66.

$1996 \mathrm{~b}$ "Cutting the Network", Journal of the Royal Anthropological Institute 2 (3) : 517-535.

1999a "The Ethnographic Effect I", in Property, Substance and Effect.

Anthropological Essays on Persons and Things. London-New Brunswick, Athlone Press : 1-26.

1999b "The Ethnographic Effect II ", in Property, Substance and Effect.

Anthropological Essays on Persons and Things. London-New Brunswick, Athlone Press: 229-261.

2000a «Afterword: Accountability... and Ethnography », in Marilyn Strathern, ed., Audit Cultures. Anthropological Studies in Accountability, Ethics and the Academy. London-New York, Routledge : 279-304.

2000b «Introduction: New

Accountabilities", in Marilyn Strathern, ed., Audit Cultures. Anthropological Studies in Accountability, Ethics and the Academy. London-New York, Routledge : 1-18.

2002 "Foreword: Not Giving the Game Away ", in Andre Gingrich \& Richard G. Fox, eds, Anthropology, by Comparison. London, Routledge : XIII-XVII. 2003a Commons and Borderlands.

Working Papers on Interdisciplinarity,

Accountability and the Flow of Knowledge.

Oxon, Sean Kingston.

2003b "Endnote: Re-describing Society", in Commons and Borderlands... : 87-102.

2004 [1991] Partial Connections.

Walnut Creek, Altamira Press.

2005a "Prologue ", in Mark S. Mosko

\& Frederick H. Damon, eds,

On the Order of Chaos. Social Anthropology

and the Science of Chaos. New York,

Berghahn : XII-XV.

2005b Kinship, Law and the Unexpected.

Relatives Are Always a Surprise. New York,

Cambridge University Press.

2006 "A Community of Critics?

Thoughts on New Knowledge", Journal of the Royal Anthropological Institute 12 (1) : 191-209.

2011a "Binary License", Common Knowledge 17 (1) : 87-103.

2011b "What Politics?", Common Knowledge 17 (1) : 123-127.

2012 «Response: A Comment on "the Ontological Turn" in Japanese Anthropology ", Hau. Journal of Ethnographic Theory 2 (2) : 402-405.

2014a "Kinship as a Relation", L'Homme 210 : 43-61.

2014b "Reading Relations Backwards", Journal of the Royal Anthropological Institute 20 (1) : 3-19.

2014c "Anthropological Reasoning:

Some Threads of Thought ", Hau. Journal of Ethnographic Theory 4 (3) : 23-37.

2017 «At the Limits of a Certain Language: Interview with Eduardo Viveiros de Castro and Carlos Fausto ", in Ashley B.

Lebner, ed., Redescribing Relations. on Ethnography, Knowledge and Politics.

New York, Berghahn Books [à paraître].

Street, Alice \& Jacob Copeman

2014 "Social Theory after Strathern :

An Introduction ", Theory, Culture

\& Society 31 (2-3) : 7-37. 
Viveiros de Castro, Eduardo

1998 "Cosmological Deixis and Amerindian Perspectivism ", Journal of the Royal

Anthropological Institute 4 (3) : 469-488.

2003 And After-dinner Speech Given at

Anthropology and Science, the $5^{\text {th }}$ Decennial

Conference of the Association of Social

Anthropologists of Great-Britain and the

Commonwealth. Manchester, Department

of Social Anthropology, ("Manchester Papers

in Social Anthropology " 7).

2011 "Zeno and the Art of Anthropology :

Of Lies, Beliefs, Paradoxes, and Other

Truths ", Common Knowledge 17 (1) :128-145.

Viveiros de Castro, Eduardo

\& Marcio Goldman

2009 «Slow Motions : Comments

on a Few Texts by Marilyn Strathern [transl. by Ashley Lebner] », Cambridge

Anthropology 28 (3) : 23-42.

2017 "Slow Motions [Extended Remix] :

Comments on a Few Texts by

Marilyn Strathern [Transl. by Ashley B.

Lebner]", in Ashley B. Lebner, ed.,

Redescribing Relations. Strathernian

Conversations on Ethnography, Knowledge and Politics. New York, Berghahn Books [à paraître].

Weiner, James

1993 «Anthropology Contra Heidegger

Part II : The Limits of Relationship ",

Critique of Anthropology 13 (3) : 285-301.

2001 Tree Leaf Talk. A Heideggerian

Anthropology. Oxford-New York, Berg. 
Ashley Lebner, La redescription de l'anthropologie selon Marilyn Strathern. - Cet article se penche sur la vision de la description anthropologique comme redescription élaborée par Marilyn Strathern. Il éclaire non seulement la façon dont les relations animent l'anthropologie de Strathern, mais aussi la façon dont celle-ci redécrit la discipline elle-même. En effet, la redescription n'est pas un "concept " qui nous permet de "comparer" des "sociétés ». Il s'agit plutôt d'une "relation ", désignée comme telle parce qu'elle a besoin des autres pour fonctionner, et qui permet par conséquent d'envisager les connexions/distinctions interpersonnelles et conceptuelles sous un jour nouveau. Larticle examine la série de relations qui composent la redescription selon Strathern - depuis le décentrement et l'analogie jusqu'aux relations et à la politique -, soulignant d'emblée l'importance du décentrement qu'elle opère de deux termes clés en anthropologie: la société et l'individu. C'est de ce décentrement qu'émerge la créativité intellectuelle de Strathern: d'une réflexion sur la socialité et la dividualité à la critique de la comparaison classique; du déploiement de la raison analogique à son travail d'écriture en tant qu'auteur-cyborg; et du développement d'un nouveau genre anthropologique à la reconceptualisation de la politique anthropologique comme redescription des relations. En allant au cœur même de la pratique anthropologique élaborée par Strathern, en montrant comment chacune de ses analyses est reliée aux autres, cet article vise à initier un nouveau dialogue avec son œuvre. Si le style ethnographique de Strathern demeure un défi, éclairer la cohérence encore peu explorée de son projet redescriptif s'avère en effet une excellente façon d'entamer de nouvelles discussions.
Ashley Lebner, Marilyn Strathern's Redescription Strathern's view of anthropological description as redescription. As such, it not only makes visible how relations animate Strathern's anthropology, but captures how Strathern redescribes anthropology in turn. Indeed, redescription is not a "concept " that will help us "compare" "societies». Rather it is a relation, so-called because it requires others to operate, and thus helps us see interpersonal and conceptual connections/ distinctions anew. The paper explores the series of relations that comprise Strathern's redescription - from displacement and analogy to relations and politics - first emphasizing the importance of Strathern's displacement of two key anthropological terms, that of society and the individual. From that displacement, it is shown that Strathern's intellectual creativity follows: from her reflections on sociality and dividuality, to her critique of conventional comparison; from her deployment of analogical reason, to her writing as cyborgian author; and from the development of a new anthropological genre, to her reconception of anthropological politics as the redescription of relations. In going to the heart of Strathern's anthropological practice, showing how each analytic is related to the other, the piece hopes to facilitate new engagement with her work. Although the challenge of her ethnographic style will remain, elucidating the rarely-explored coherence of her project is a good way to begin new discussions. 